\title{
Live Demonstration: A Wearable EIT System Using Active Electrodes for Monitoring Respiration
}

\author{
$\mathrm{Yu} \mathrm{Wu}{ }^{1}$, Dai Jiang ${ }^{1}$, Andy Bardill ${ }^{2}$, Serena De Gelidi ${ }^{2}$, Richard Bayford ${ }^{2}$ and Andreas Demosthenous ${ }^{1}$ \\ ${ }^{1}$ Department of Electronic and Electrical Engineering, University College London, Torrington Place, London WC1E 7JE, UK \\ ${ }^{2}$ Department of Natural Sciences, Middlesex University, The Burroughs, London, NW4 4BT, UK \\ e-mail: yu.wu.09@ucl.ac.uk; a.demosthenous@ucl.ac.uk
}

\begin{abstract}
A wearable electrical impedance tomography system for lung respiratory monitoring will be demonstrated. The system features an active electrode integrated circuit (IC) fabricated in 0.35- $\mu \mathrm{m}$ CMOS technology. The IC is mounted on a flexible printed circuit board which is then embedded in a textile belt. During the live demonstration, the belt can be put on the volunteers' chest, and through a back-end signal processing hub, the system is able to provide real-time lung respiratory images on a remote computer.
\end{abstract}

\section{INTRODUCTION}

Electrical impedance tomography (EIT) is a non-invasive, and radiation-free technique that can provide real time lung function monitoring - many studies have shown that it is effective in monitoring aeration in both adult and preterm babies [1]. EIT images an object by injecting ac currents through electrodes on the object's surface. Potentials are then developed on all the electrodes and can be recorded. Algorithms use the recorded voltages to estimate the distribution of the inner conductivity (resistivity) of the object, and reconstruct an image representation.

Many studies have suggested that using active electrodes can improve the signal integrity, and provide more reliable EIT images. To overcome the challenges faced in developing an active EIT system, an active electrode integrated circuit (IC) containing a wideband current driver and low noise amplifier has been developed [2]. An EIT system using this IC and featuring a wearable belt with embedded active electrodes has been developed. The IC is mounted on the orange flexible printed circuit board (FPCB) as shown in Fig.1 (a), and the FPCB is wrapped within a white self-adhesive textile belt cover. Silver-coated fabric electrodes are attached on both the inner and outer layers of this belt cover, connecting the active electrode to the test subject. Through a cable, the active belt is then connected back to the control and signal processing hub where the excitation signal for the current driver is generated and the recording voltage is digitized and sent to a remote computer for image reconstruction.

\section{DEMONSTRATION SETUP AND Visitor EXPERIENCE}

The proposed demonstration setup includes the wearable active belt, the back-end control and signal processing hub as well as a remote laptop computer. After applying a body moisturiser cream, a volunteer can wear the belt around the chest as shown in Fig.1 (b).

This work is funded by the European Commission under grant agreement no. 668259 - CRADL (www.cradlproject.org).

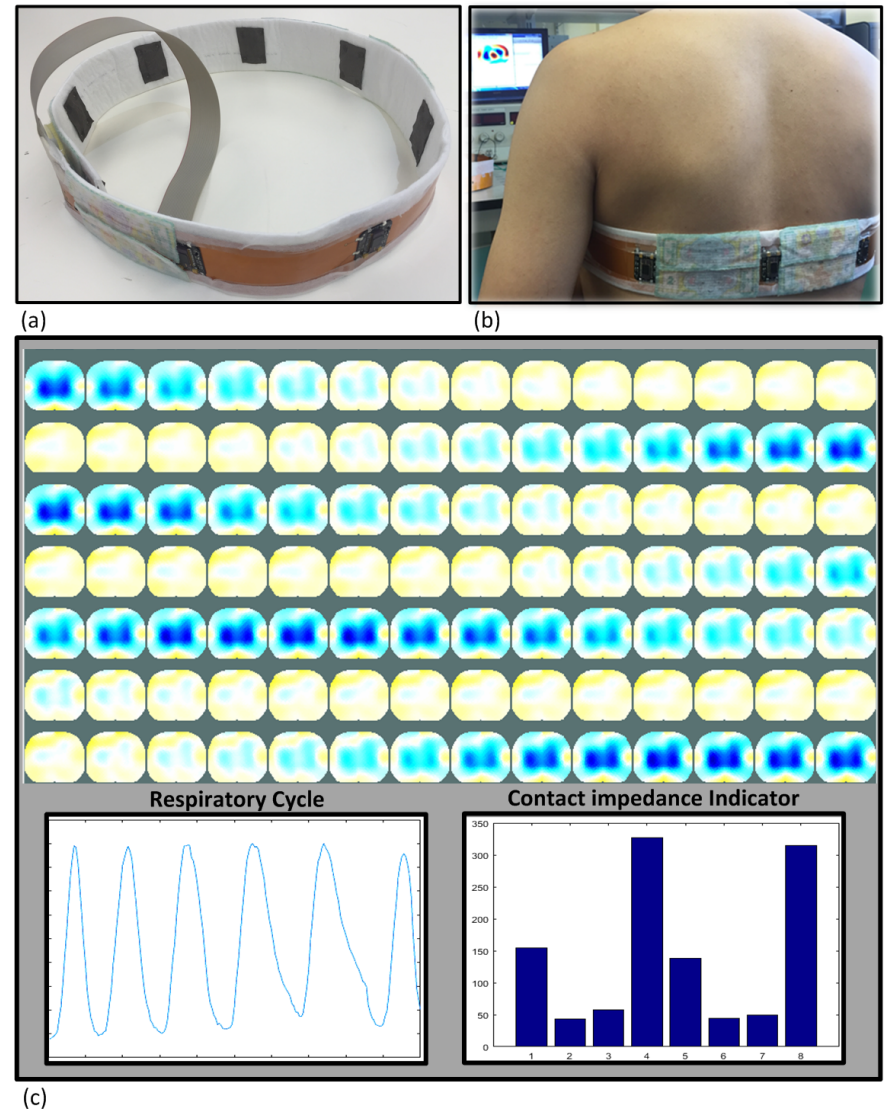

Fig.1. (a). Wearable EIT system using active electrodes with embedded ICs. (b). System worn by a volunteer. (c). GUI shows the lung respiration images, and breathing cycles while monitoring the electrode contact conditions.

A custom made graphical user interface (GUI) will run on the laptop to produce lung respiratory images in real-time. The breathing cycles and the electrode contact conditions can also be displayed as shown in Fig.1 (c).

Visitors will be able to either observe the live demonstration of our own volunteer, or to participate in wearing the belt and observe their own respiratory cycles.

\section{REFERENCES}

[1] B.Gong, S, Krueger-Ziolek, et al. "Electrical impedance tomography: functional lung imaging on its way to clinical practice?" Expert Review of Respiratory Medicine, 9:6 , 721-737

[2] Y. Wu, P. Langlois, R. Bayford and A. Demosthenous, "Design of a CMOS active electrode IC for wearable electrical impedance tomography systems," 2016 ISCAS, Montreal, QC, 2016, pp. 846-84 